\title{
Solar Energetic Particles and Solar Events - Lessons Learned from Multi-Spacecraft Observations
}

\author{
Christina M.S. Cohen ${ }^{1}$ \\ California Institute of Technology \\ MC 290-17, Pasadena, CA, USA \\ E-mail: cohenesrl.cal tech.edu
}

\begin{abstract}
Never before has the heliosphere and the Sun been so carefully monitored by so many spacecraft; in particular, the STEREO spacecraft have allowed simultaneous observations to be made routinely from multiple solar longitudes. The instrumentation on these spacecraft are continually observing solar activity and measuring the characteristics of solar energetic particle (SEP) events, providing a wealth of information on the acceleration and transport of SEPs. In February, 2011 the STEREO spacecraft reached a separation of $180^{\circ}$ and since then the entire solar surface has been visible. This unprecedented view has allowed observations of active regions and solar activity to continue after a region has rotated over the limb (as view from Earth) and more importantly, of regions emerging on the solar hemisphere not visible to Earth. The multiple viewpoints afforded by spectrometers and coronagraphs on the STEREO and near-Earth spacecraft has yielded more accurate information regarding the speed, direction, and evolution of coronal mass ejections (CMEs) which drive the interplanetary shocks that generate large SEP events. As is often the case when new capabilities are achieved or new regimes are explored, even while some questions are answered, more emerge. Among the surprises from multi-spacecraft SEP observations is the exceptionally fast longitudinal transport of particles. This paper reviews these multi-spacecraft capabilities, highlights some of the recent observations and surprises, and discusses the impact on the current understanding of energetic particle acceleration and transport.
\end{abstract}

The 34th International Cosmic Ray Conference

30 July- 6 August, 2015

The Hague, The Netherlands

\footnotetext{
${ }^{1}$ Speaker 


\section{Introduction}

The protection of space-based assets as well as various communication systems on Earth, the electrical power grid, and managing radiation exposure of commercial airline crews all require accurate space weather forecasting and now-casting. Characterizing and understanding the different aspects of space weather is a critical step towards that developing that ability. Until recently, connecting the source of space weather (e.g., remotely observed activity on abd near the Sun) with the resulting hazards (e.g., energetic particle, interplanetary shock, and geomagnetic storm characteristics measured in-situ) has been hampered by the limited observations well away from the Earth, e.g., at different solar longitudes and/or at distances closer to the Sun. Although the world has numerous scientific spacecraft currently operating, the vast majority of these are making measurements in the Earth's magnetosphere or at the L1 Lagrangian point along the Sun-Earth line.

The difficulties in linking the origins of solar energetic particle (SEP) events with the particle characteristics using data from only near-Earth observations is illustrated in Figure 1. With a single observation point, the longitudinal dependence of any SEP event characteristic, e.g., the $\mathrm{Fe} / \mathrm{O}$ abundance ratio, can only be studied in a statistical sense by examining the position of the solar source regions relative to the Sun-Earth line (defined as $0^{\circ}$ solar longitude) for a large number of events. Figure 1 shows 86 SEP events, each plotted at the solar longitude of the source and the measured $\mathrm{Fe} / \mathrm{O}$ abundance ratio (normalized to 0.134 , the average SEP value obtained in a previous study [2]). Determining whether a longitude dependence exists, is difficult from such a plot (and opinions differ, c.f. [3] and [4]) as each event is subject to different solar and interplanetary conditions affecting the acceleration and the transport of the particles and ultimately the measured $\mathrm{Fe} / \mathrm{O}$ ratio. To accurately determine the longitude dependence measurements at distinctly different longitudes need to be made simultaneously within individual events.

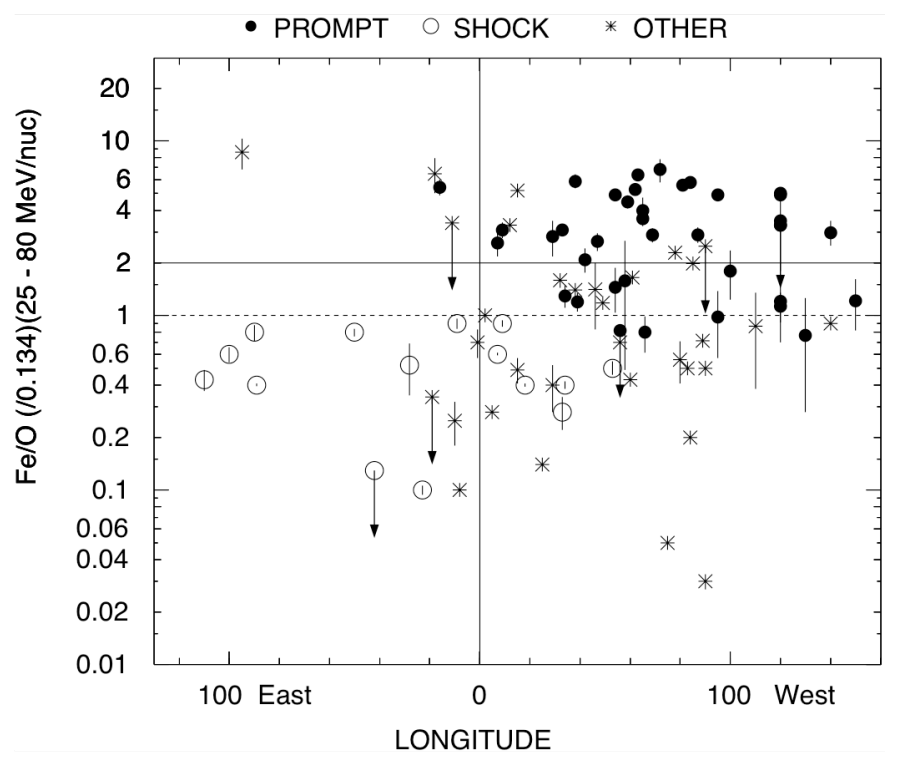

Figure 1: The Fe/O ratio (normalized to 0.134 ) measured at $25-80 \mathrm{MeV} /$ nuc as a function of the solar longitude of the solar source for 86 SEP events. The Sun-Earth line at $0^{\circ}$ is marked with a vertical line. From [1]. 
Exactly this capability was obtained with the launch of the twin Solar Terrestrial Relations Observatory (STEREO) spacecraft in October 2006. It is now possible to routinely make simultaneous in-situ measurements of the interplanetary plasma and the energetic particle population, as well as view the Sun remotely in different wavelengths and with coronagraphs, at multiple solar longitudes. The mission consists of two identical spacecraft orbiting the Sun at $\sim 1 \mathrm{AU}$, one (STEREO-A) slightly faster than the Earth and the other (STEREO-B) slightly slower. The difference in orbital periods results in the separation between each spacecraft and the Sun-Earth line increasing at a rate of $\sim 22.5^{\circ}$ year (Figure 2) [6]. The addition of the STEREO spacecraft to the scientific fleet has resulted in new capabilities for studying SEP events and the related solar activity. This paper highlights these and reviews the associated scientific results. As is often the case when exploring a new regime, in answering existing questions, new ones arise; these will also be discussed.
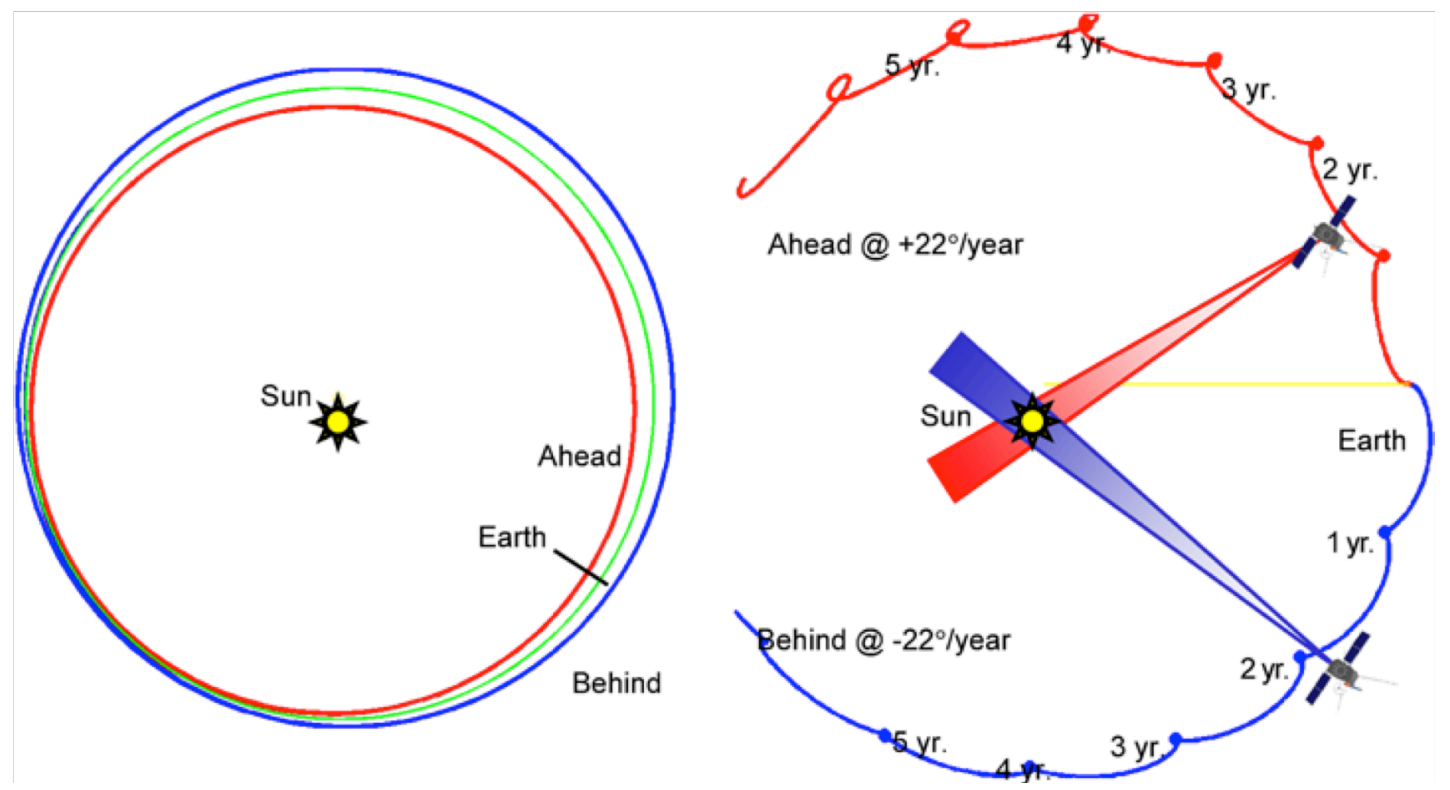

Figure 2: (left) The orbits of the twin STEREO spacecraft (red for STEREO-A, blue for STEREO-B); (right) the orbits relative to the Sun-Earth line. From [5].

\section{Multi-spacecraft capabilities}

The SEP instrumentation on the STEREO spacecraft [7] has similar energy and species coverage to the sensors on the Advanced Composition Explorer (ACE) spacecraft [8]. This allows quantitative comparisons to be made between the observations obtained by each spacecraft. For SEP events detected at STEREO and ACE, the longitudinal dependence of the SEP characteristics can be directly determined. Additionally, this dependence can be compared between events to extract more information regarding the variability of the factors affecting particle transport through the interplanetary medium.

Not surprisingly, studies of the solar activity related to SEP events also benefit significantly from multi-point observations. By measuring the position of a coronal mass ejection (CME) as it moves through the field of view of a coronagraph as a function of time, the velocity of the $\mathrm{CME}$ can be calculated. However, from a single viewpoint, only the velocity component in the plane of the observed sky is determined. For CMEs traveling orthogonally 


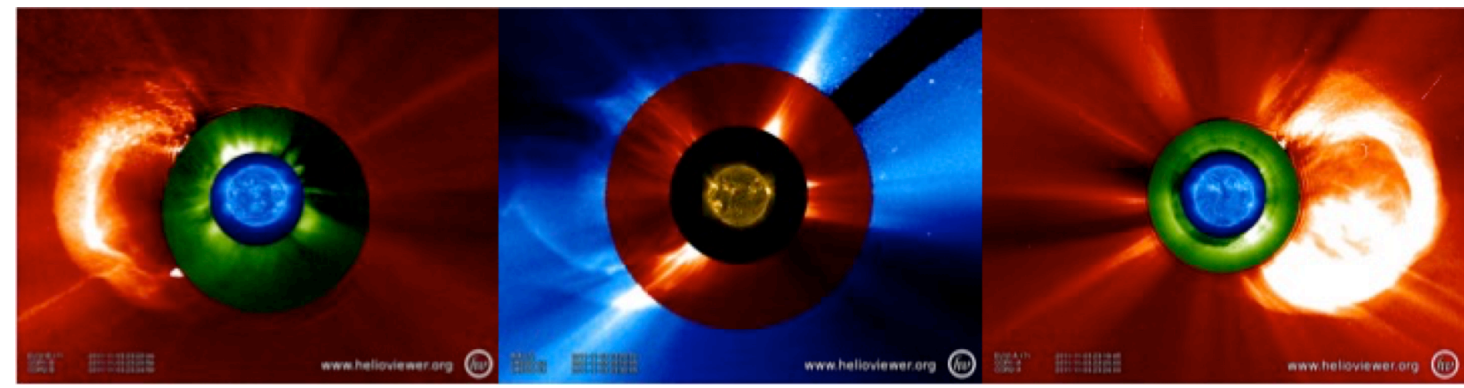

Figure 3: Images of a CME from (left) STEREO-B, (center) SOHO (with SDO inset), and (right) STEREO-A. The direction of the CME seen by both STEREOs make it clear that although this was a halo CME seen from Earth it was heading away from Earth.

relative to the viewer, the plane-of-sky speed is close to the total speed; however, for events moving directly towards the observer (called 'halo CMEs') the measured speed can be significantly lower than the true speed; additionally it is ambiguous as to whether the CME is heading towards or away from the observer. By combining the observations of the same CME from different vantage points a more accurate calculation of the speed can be made. Figure 3 shows the views from both STEREOs and the Solar Heliospheric Observatory (SOHO) at L1 of a CME seen as a halo from Earth. The observations from the STEREO coronagraphs reveal that the CME was traveling away from Earth. Sophisticated modeling techniques have incorporated data such as these to create 3D representations of the CME and its evolution, providing more detailed information on its position, size, and development than can be gleaned from the data obtained at a single location (e.g., [9]).

Once the separation between each STEREO spacecraft and the Earth reached $90^{\circ}$, in February 2011, the entire surface of the Sun was visible. This allowed the solar source of any SEP event to be readily identified. Prior to this, only sources on the Earth-facing side of the Sun could be examined in detail. While some 'over-the-limb' sources were identified in past SEP studies, using data of flaring active regions from several days before the SEP event and

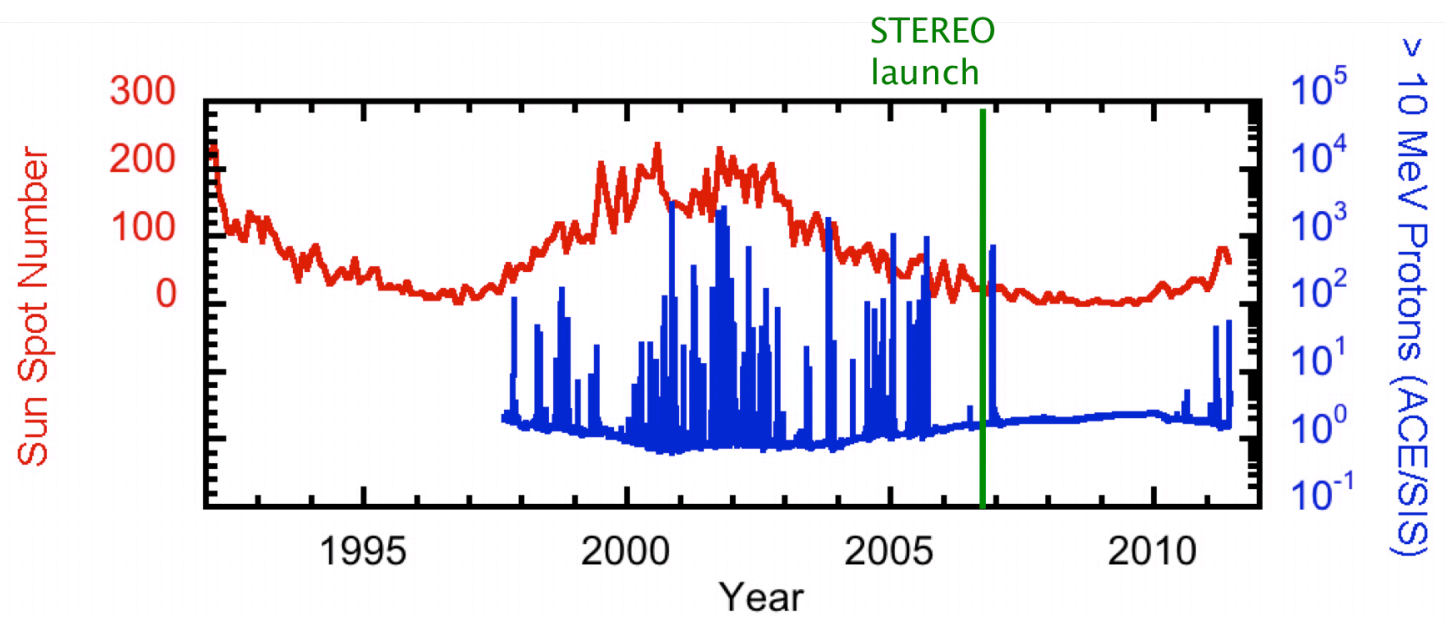

Figure 4: Sunspot number (red, left axis) and $>10 \mathrm{MeV}$ proton intensities, measured by ACE/SIS (blue, right axis) versus time. The green vertical line indicates the launch date of STEREO. The large spikes in the $>10 \mathrm{MeV}$ proton intensity profile are SEP events. 
ISEE-3

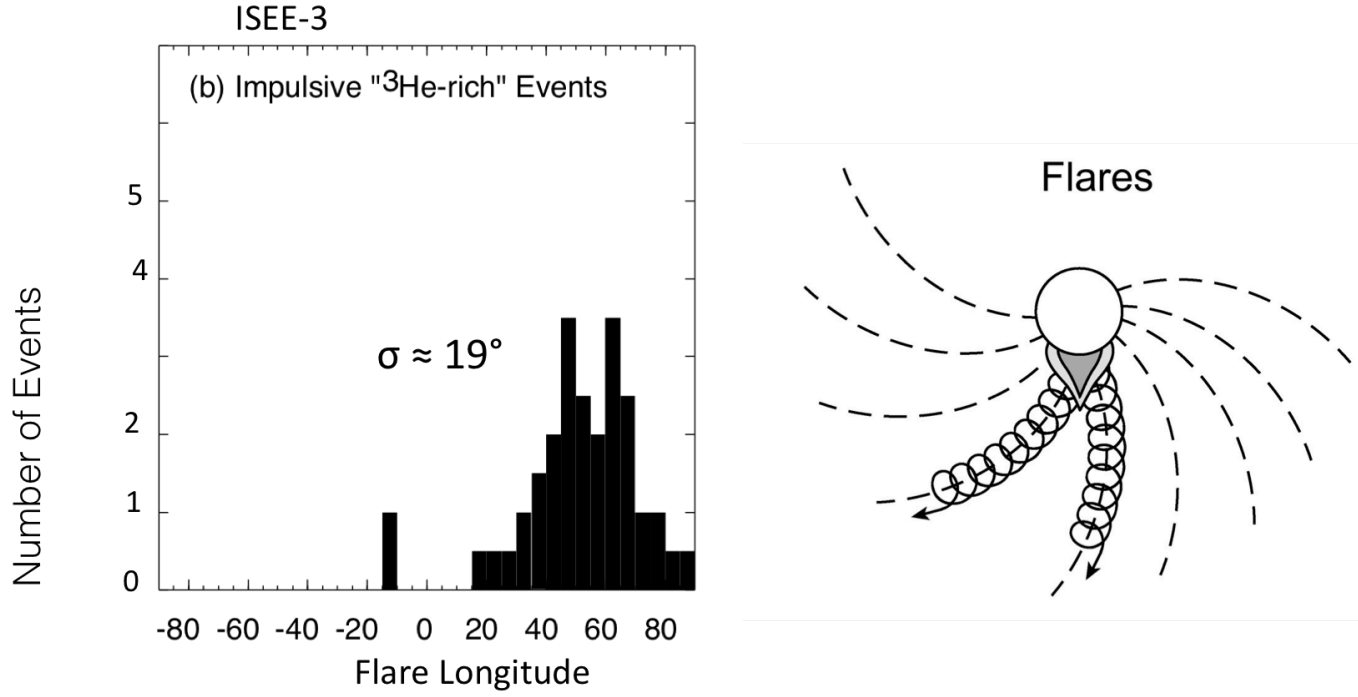

Figure 5: (left) Histogram of source region longitudes for ${ }^{3} \mathrm{He}$-rich SEP events showing the clustering around the magnetically well connected longitude of $\sim 60^{\circ}$ for a nominal Parker spiral. (right) Cartoon showing the corresponding understanding that particles accelerated in the flaring region are injected onto a set of field lines that are only narrowly spread in longitude. From [2].

estimating where the active region would be (after rotating out of view) when the SEP event occurred, the characteristics of the flares and regions were not known. Further, regions which emerged on the solar hemisphere not visible from Earth could result in SEP events without an identified source, or in some cases, an incorrectly selected source on the Earth side of the Sun.

\section{3. $\quad{ }^{3}$ He-rich SEP Events}

Unfortunately STEREO was launched into the quietest solar minimum in decades. Figure 4 shows the launch date in the context of the cycle evolution of the sunspot number and the intensity of $>10 \mathrm{MeV}$ protons. Aside from the two large SEP events which occurred in December 2006, shortly after launch, the STEREO spacecraft did not observe any SEP events of
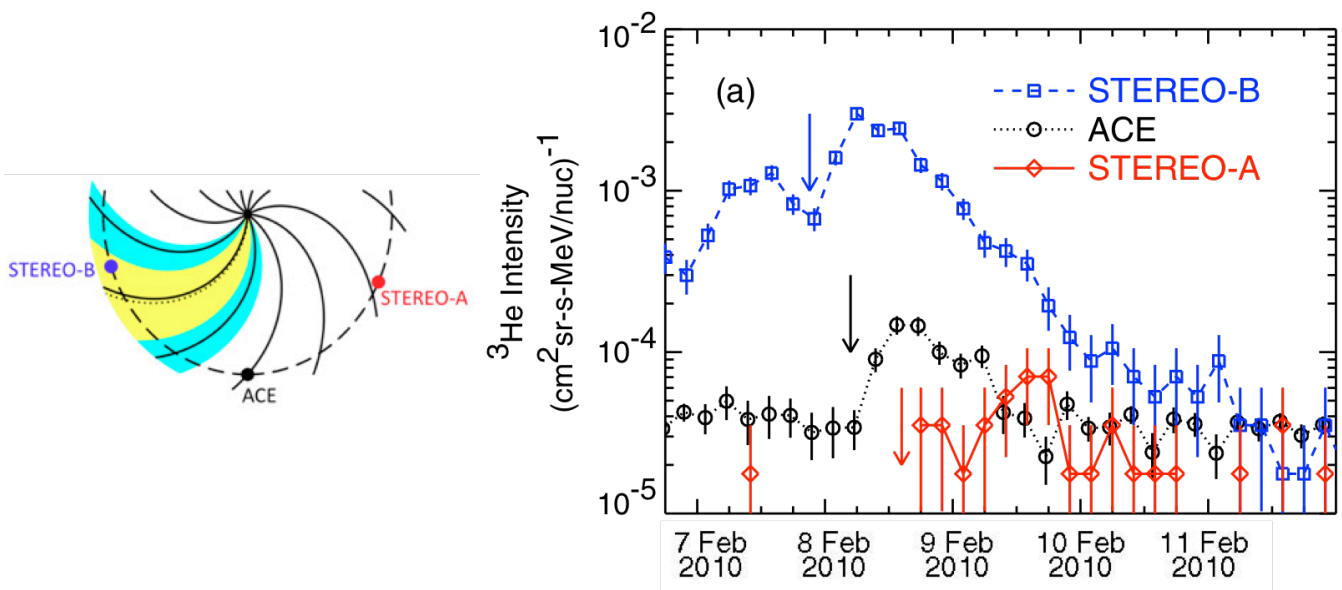

Figure 6: (left) Positioning of ACE and the two STEREO spacecraft in February 2010. The shaded regions indicate the expected $1-\sigma$ (yellow) and $2-\sigma$ (blue) spread around the magnetic connection to the source region (located near disk center as observed from Earth). (right) The ${ }^{3} \mathrm{He}$ intensities observed by the three spacecraft; all three spacecraft registered the event despite their wide separation. Colored arrows indicate the approximate onset times of the event at each spacecraft. From [10]. 

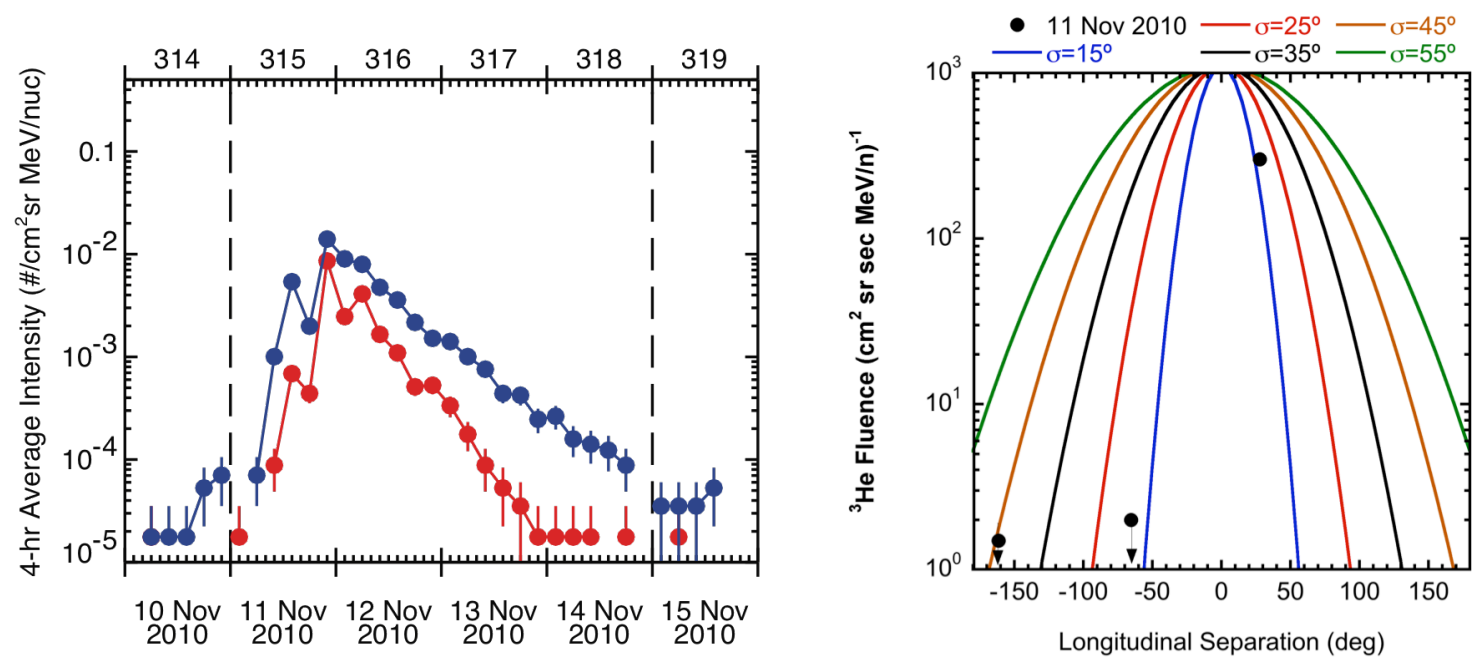

Figure 7: (left) Time profiles of ${ }^{3} \mathrm{He}$ (red) and ${ }^{4} \mathrm{He}$ (blue) for the single spacecraft ${ }^{3} \mathrm{He}$-rich event of 11 November 2010 as observed by STEREO-B. (right) The event-integrated ${ }^{3} \mathrm{He}$ fluence from STEREO-B (upper point) and upper limits determined from ACE and STEREO-A data (points with downward arrows) as a function of the distance between the flaring region and the magnetic connection point of the spacecraft (assuming a Parker spiral). Curves correspond to Gaussian distributions centered on the location of the solar flaring region for various widths. Only a Gaussian with a width less than $\sim 15^{\circ}$ is consistent with the data. From [11].

significant size until 2011. However, there was an advantage to the extremely quiet solar conditions; the study of smaller, ${ }^{3} \mathrm{He}$-rich SEP events was possible due to the lower SEP background and often the source region of these events was easily identified as it was the only flaring region on the Sun [10]. Historical studies of these events (see e.g., [2]) indicated that an observer must be well connected magnetically to the source region to detect ${ }^{3} \mathrm{He}$-rich events. The associated interpretation is that these events primarily consist of particles accelerated in the flaring region and injected onto a narrow longitudinal region of open field lines (Figure 5).

Thus it was quite a surprise when a ${ }^{3} \mathrm{He}$-rich SEP event in February 2010 was seen by ACE and both STEREO spacecraft, which were separated by $136^{\circ}$ (Figure 6; [10]). The event was most clearly seen by STEREO-B which was magnetically well connected to the source, an active region located near disk center as viewed from Earth. The histogram shown in Figure 5 has a sigma of $\sim 19^{\circ}$ in longitude; taking this as typical for ${ }^{3} \mathrm{He}$-rich events, ACE would not be expected to measure the 7 February 2010 event as it was $\sim 70^{\circ}$ to the west of STEREO-B. However, the event was clearly seen by ACE and even by STEREO-A which was another $65^{\circ}$ further west. The quiet solar conditions allowed clear confirmation that all three spacecraft were measuring the same SEP event (no other active region on the Sun was flaring at the appropriate time).

As unusual as the 7 February 2010 event seemed, a survey of SEP data from sensors on STEREO and ACE revealed many events with spreads of $>60^{\circ}$ (as measured by 2 or more spacecraft) [12]. This raises the question as to whether all ${ }^{3} \mathrm{He}$-rich events are longitudinally wide and previous studies simply did not have the sensitivity to determine the true spread. An effort to study this possibility was made by selecting ${ }^{3} \mathrm{He}$-rich events of similar peak intensity to that of the 7 February 2010 event (Figure 7, left panel), yet observed by only one of the STEREO spacecraft and not by the other or by ACE [11]. Using the ${ }^{3} \mathrm{He}$ fluence observed by 


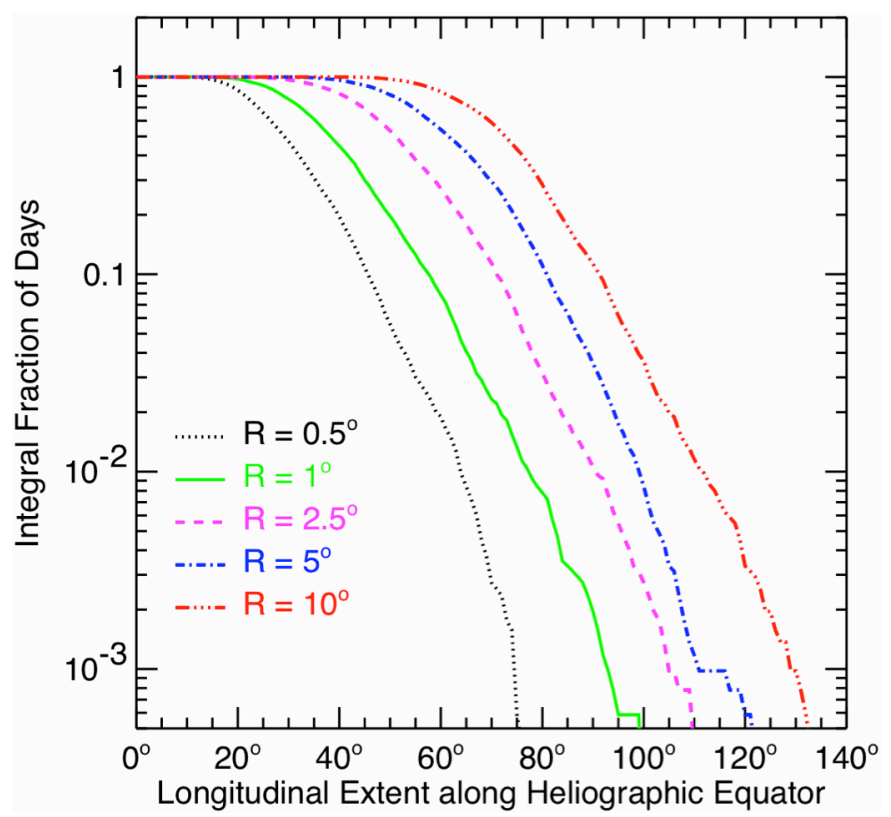

Figure 8: The integral fraction of days for which a given longitudinal spread at $2.5 \mathrm{R}_{\mathrm{S}}$ can result from a photospheric source of radius $0.5^{\circ}-10^{\circ}$ (different lines). From [10].

one spacecraft and the upper limits derived from the other two spacecraft and assuming a Gaussian distribution centered on the location of the flaring region, the authors derived the maximum width in longitude consistent with the observations (Figure 7, right panel). The results indicated that the 'single-spacecraft' events were significantly narrower in longitudinal spread than the multi-spacecraft events identified in previous studies [10] making it clear that only some ${ }^{3} \mathrm{He}$-rich events are wide. Thus the cause of the wide spreads must be a process that, while not ubiquitous, is also not rare.

\section{Causes of wide 3He-rich SEP events}

Several possibilities for the generation of wide ${ }^{3} \mathrm{He}$-rich SEP events have been put forth including:

1. Multiple solar sources

2. Field line spreading from the photosphere to the corona

3. Complex reconnection near the Sun

4. Field line meandering in the interplanetary medium

5. Field line co-rotation

6. Cross-field diffusion near the Sun and/or in the interplanetary medium

Item 1 has been generally ruled out, aided by the quiet solar conditions in which the observations have been made and item 4 is believed to create only a $\sim 5-10^{\circ}$ spread ( $\mathrm{J}$. Giacalone, private communication 2014), not enough to explain the observations. Item 2 has been examined by Wiedenbeck et al. [10] by using 14 years of daily Potential Field Source Surface (PFSS) maps of field line spreads between the photosphere and $2.5 \mathrm{R}_{\mathrm{S}}$. The authors determined the largest longitudinal spread at the heliographic equator at $2.5 \mathrm{R}_{\mathrm{S}}$ resulting from a bundle of magnetic field lines originating from photospheric regions with radii between $0.5^{\circ}$ and $10^{\circ}$. The resulting integral fraction of days for a given longitudinal spread is given in Figure 8 . 


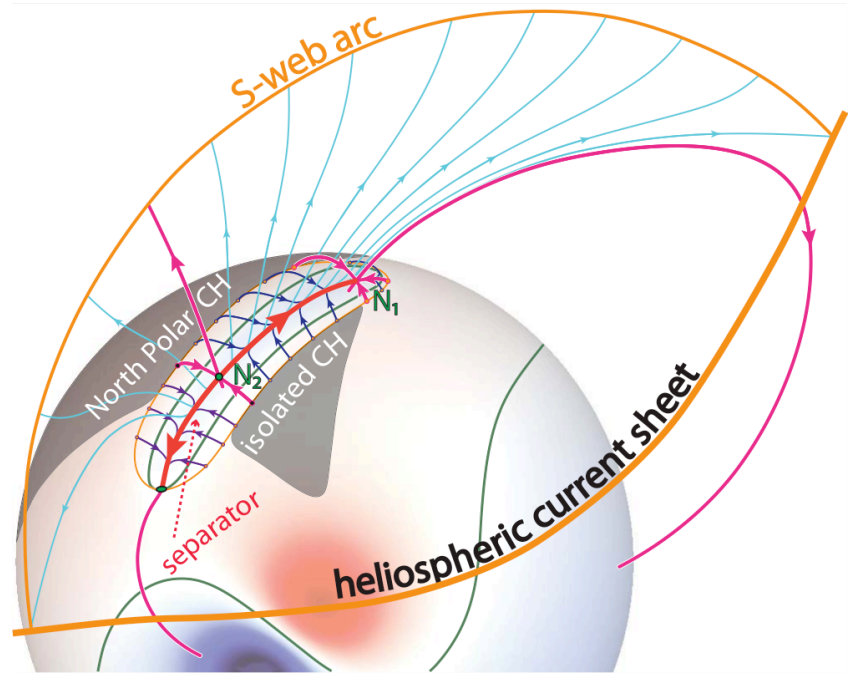

Figure 9: An idealized source-surface model of the corona for a dipole plus a bipolar active region, rotated for a better view of the isolated coronal hole. The magnetic flux on the photosphere is shown as a blue-red color map ( $-\mathrm{Br}$ blue, $+\mathrm{Br}$ red), together with open field regions (shaded in gray). Polarity inversion lines are shown in green. Field lines along the S-web arc all map to a single null point (N2) allowing for a wide distribution of particles originating from near N2. Adapted from [13].

It can be seen that a spread of $>120^{\circ}$, such as was seen in the 7 February 2010 event, is extremely rare, thus item 2 is unlikely to be the explanation for wide ${ }^{3} \mathrm{He}$-rich SEP events.

The heliospheric impact of complex magnetic topologies on the Sun (item 3) is being studied through sophisticated modeling and theoretical efforts (see e.g., [14]). Recently it has been noted that these topologies may have significant implications for particle distribution through magnetic reconnection [15]. Figure 9 illustrates how emersion of a region of parasitic polarity, isolating a section of a polar coronal hole, can result in a magnetic separator which connects a single null point near the photosphere to a separatrix web (S-web) arc in the corona which can span large angles [13]. Simulations based on photospheric magnetograms obtained during the times of wide and narrow ${ }^{3} \mathrm{He}$-rich events are being compared to determine if the characteristics and/or presence/absence of an S-web distinguishes between the two types of ${ }^{3} \mathrm{He}-$ rich SEP events. So far the results are incomplete but encouraging (J. Linker, private communication 2015).

It has been noticed that the onset times for the three spacecraft in the 7 February 2010 event spanned nearly a day (Figure 6); during this time the Sun would have rotated a significant amount causing the interplanetary field lines to be convected westwards, carrying particles with them. The contribution of this corotation to the observed particle longitude spread was examined with simulations by Giacalone and Jokipii [16]. With reasonable assumed levels of perpendicular diffusion, the authors were able to match the longitudinal spread of the ${ }^{3} \mathrm{He}$ fluences. Depending on the chosen values of the parallel mean free path of $\sim 1 \mathrm{MeV} / \mathrm{nuc}$ particles and the ratio of perpendicular to parallel diffusion coefficients, the theory yields onset delay times (relative to the solar flare time) that increase with increasing longitude separation from longitude of best magnetic connection to the source, i.e., $\sim 60^{\circ}$ (Figure 10, left panel). To test the theory, Cohen et al. [17] examined the observed onset times as a function of longitude for $17{ }^{3} \mathrm{He}$-rich, multi-spacecraft events (Figure 10, right panel). While some events showed increased delays (relative to the flare time) with increasing longitude separation, some events 

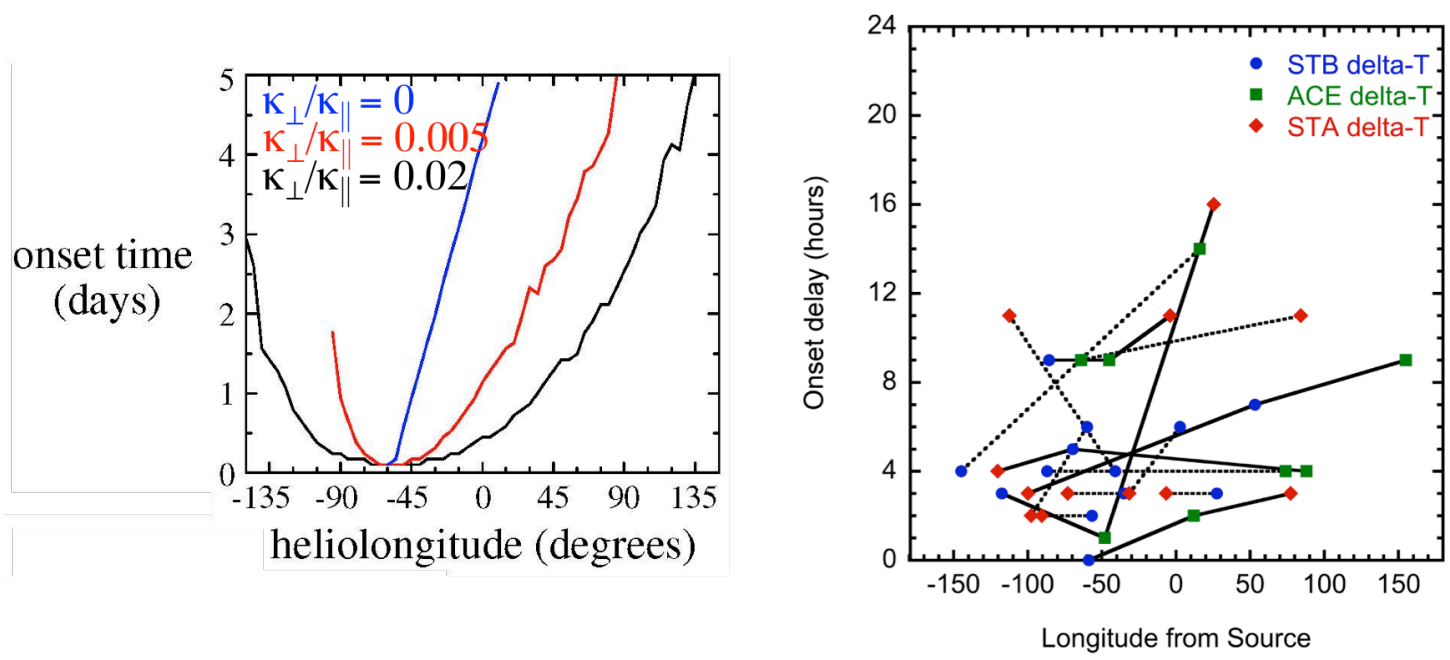

Figure 10: (left) Onset times (relative to the flare time) as a function of heliolongitude resulting for different assumptions of the ratio of perpendicular to parallel diffusion and a parallel mean free path length of $0.52 \mathrm{AU}$. The minimum delays correspond to a heliolongitude of $60^{\circ}$ where the observer is magnetically connected directly to the solar source region. From [16]. (right) Observed onset times (relative to the flare time) for 17 multi-spacecraft ${ }^{3} \mathrm{He}$-rich SEP events. Values from three-spacecraft events are connected by solid lines, while two-spacecraft events are connected by dashed lines. From [17]. Note the difference in scale (units and magnitude) between the two figures.

showed minimal longitude dependence. Additionally, the majority of the onset delay times were $<12$ hours, even for longitude separations more than $>45^{\circ}$ from best connection, while the theory predicts delays of $\geq 1$ day for such separations, for all combinations of the mean free path and diffusion parameters studied. This suggests that item 5 can be eliminated as the primary cause of wide ${ }^{3} \mathrm{He}$-rich SEP events.

The role of cross-field diffusion (item 6) in the transport of particles has recently been examined by Dresing et al. [18]. Although the study was of wide energetic electron events rather than ${ }^{3} \mathrm{He}$-rich SEP events, the results are probably relevant for both. The advantage of examining electron events is that measurements of the particle anisotropy by multiple spacecraft are readily available and can be related to the amount of scattering the particles experience during transport. The authors found that the wide events could be generally classified into one of three categories based on their anisotropies and intensities observed as a function of longitude; these are illustrated in Figure 11. In the simplest case, the longitudinal extent of the solar source region is taken to be small and the event is most intense and exhibits high anisotropies at the spacecraft best connected magnetically to that region. The other two categories required a more extended source near the Sun to explain high intensities and high anisotropies at locations less well connected to the flaring source region. This extended source was assumed to be a result of particle transport near the Sun, although the authors were not able to distinguish between different processes such as coronal transport and acceleration at an extended CME-related coronal shock. In the most complicated events, regions of high anisotropy were intermixed with regions of medium anisotropy reflecting interplanetary conditions that varied significantly in longitude. The study concluded that extended particle distributions in longitude are often a result of cross-field diffusion in the interplanetary medium as well as sometimes in conjunction with processes closer to the Sun, giving support for item 6. 

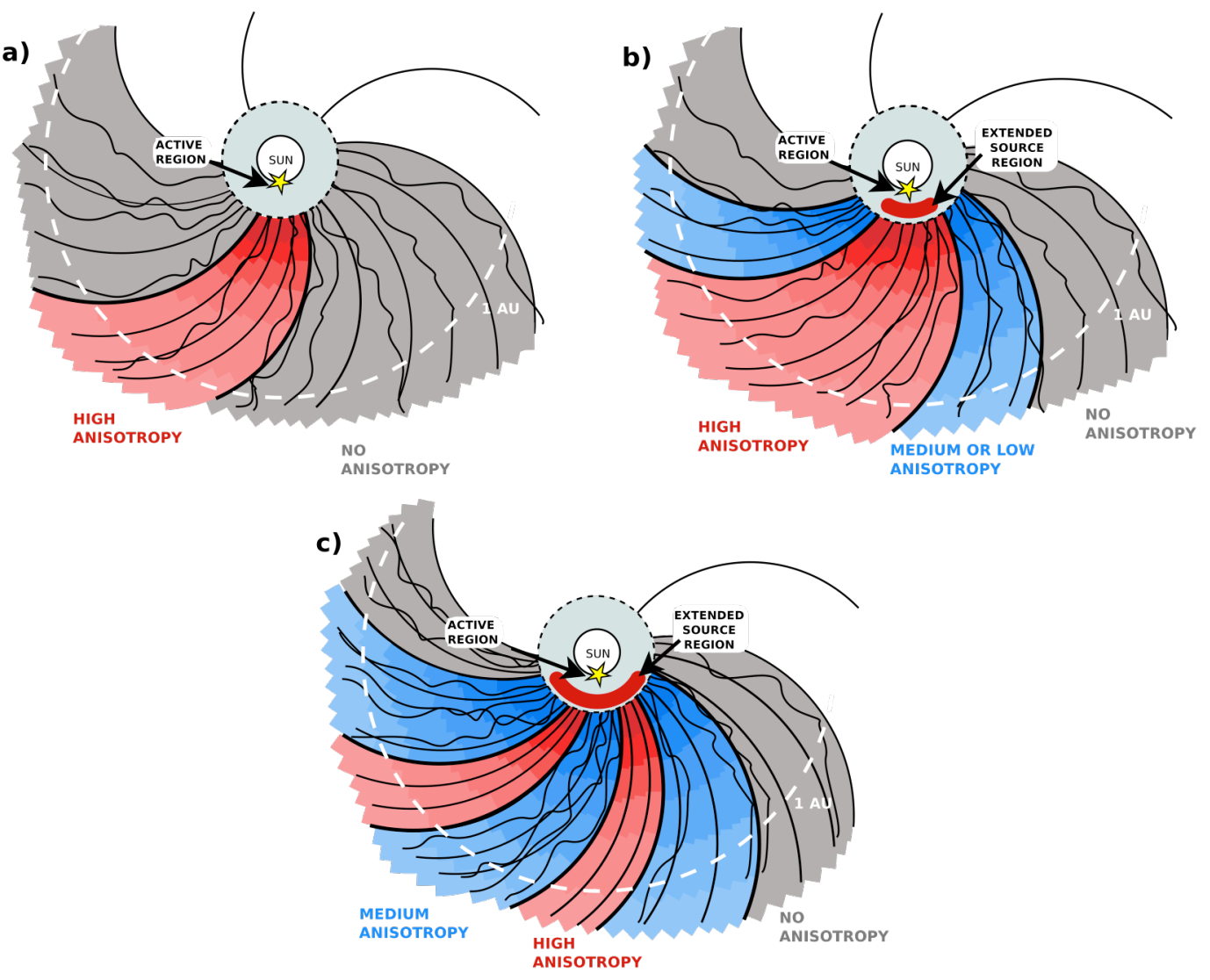

Figure 11: Cartoons depicting the different kinds of wide energetic electron events observed. Panel a) involves a small compact source (indicated by the star), while panels b) and c) have extended source regions (indicated by the red arc). The degree of observed anisotropy is indicated by the colored regions (red for high, blue for medium and grey for low/none). Wavey black lines indicate regions of perpendicular diffusion in the interplanetary medium. From [18].

The surprising discovery of wide ${ }^{3} \mathrm{He}$-rich SEP events was one of the first to result from multi-spacecraft SEP observations. While a number of possible explanations have been effectively eliminated, a definitive explanation has yet to emerge. The recent studies suggest that the relevant processes include perpendicular diffusion in the interplanetary medium and/or close to the Sun and perhaps complex magnetic structures and reconnection near the solar source region. However, much work remains before a full understanding will be achieved.

\section{Large SEP Events}

As the solar activity increased (from $\sim 2011$ onwards), it became harder to study the small ${ }^{3} \mathrm{He}$-rich events and unambiguously identify their solar sources. Naturally, the focus of multispacecraft studies turned towards the increasingly more frequent and larger SEP events associated with fast and wide CMEs. Although the acceleration mechanism for these events (diffusive shock acceleration at the CME-driven shocks) is relatively well understood (e.g., $[19,20])$, multi-spacecraft observations still yielded surprising results.

By this time, the separation of the STEREO spacecraft was such that the entire solar surface was monitored and the solar source region for any large SEP event could be readily identified. Although it was known that occasionally large SEP events observed by spacecraft near Earth had sources beyond either the east or west limb as viewed from Earth, prior to multi- 


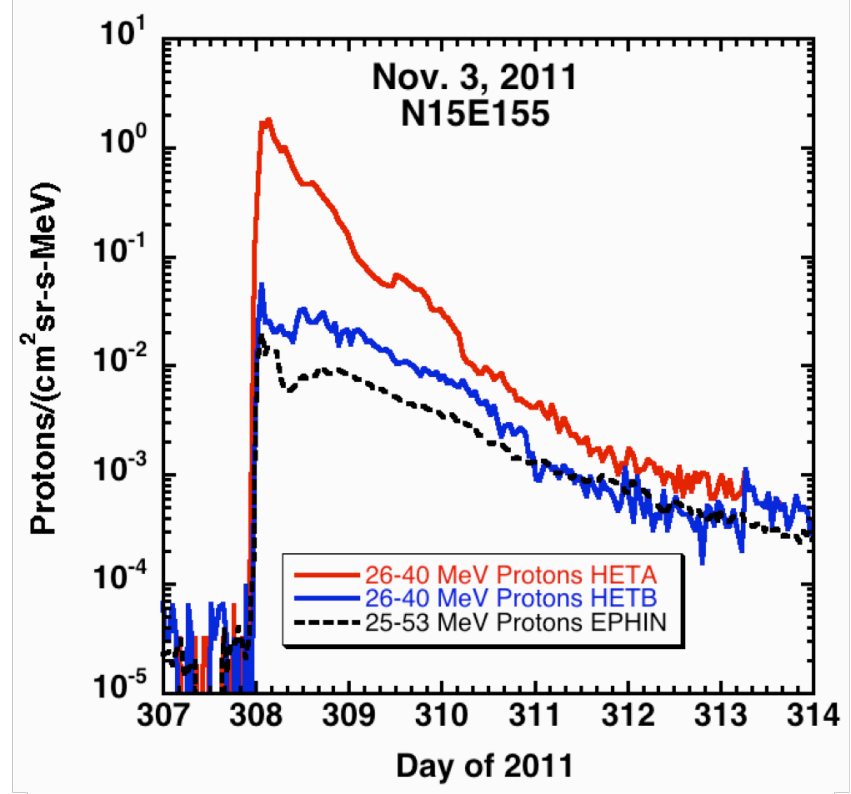

Figure 12: Energetic proton intensities versus time for the 3 November 2011 SEP event as observed by the HET instrument on the two STEREO spacecraft and the EPHIN instrument on SOHO. Although the source was located $155^{\circ}$ east of the Sun-Earth line, the particle onsets at all three spacecraft occur within 25 minutes of each other indicating very efficient distribution of particles $360^{\circ}$ around the Sun. From [22].

spacecraft observations it was difficult to accurately determine the location of such over-thelimb sources and impossible if the region had emerged on the non-Earth side of the Sun. A study of SEP events with measureable $>25 \mathrm{MeV}$ proton intensities found that $\sim 30 \%$ of the events had sources beyond the limb of the Sun as view by the observer [21]. Such a large percentage is a clear concern for space weather prediction if only near-Earth solar monitors are relied on.

Typically the spacecraft which were better magnetically connected to the source region saw the largest SEP intensities and the earliest event onsets [21]. However, a number of cases, revealed surprisingly fast transport of SEPs over $360^{\circ}$. Figure 12 shows the $\sim 25-40 \mathrm{MeV}$ proton intensities observed by both STEREOs and SOHO for the 3 November 2011 SEP event. Despite the STEREO spacecraft being $\sim 100^{\circ}$ separated from Earth and the source region being located $155^{\circ}$ east of the Sun-Earth line, the particle onsets at all three spacecraft occurred within 25 minutes of each other [21,22]. Such rapid onsets at locations well removed from the source region are in opposition to expectations [23] and again underscore how even sources invisible from Earth can be significant space weather concerns for near-Earth assets.

The National Oceanic and Atmospheric Administration (NOAA) maintains a list of SEP events that have affected the Earth since 1976 as measured by the Geostationary Operational Environmental Satellites (GOES) (http://legacy-www.swpc.noaa.gov/ftpdir/indices/SPE.txt). An event is defined when the $>10 \mathrm{MeV}$ GOES proton fluxes exceed 10 particle flux units (pfu=particles $/ \mathrm{cm}^{2}$-sec-sr) for three successive 5-minute measurements. NOAA further classifies the severity of the event by the proton peak flux: $10-100 \mathrm{pfu}=$ Minor; $100-1000 \mathrm{pfu}=$ Moderate; $10^{3}-10^{4} \mathrm{pfu}=$ Strong; $10^{4}-10^{5} \mathrm{pfu}=$ Severe; $>10^{5} \mathrm{pfu}=$ Extreme. Since 1976 there have been nine Severe SEP events, but no Extreme events. 


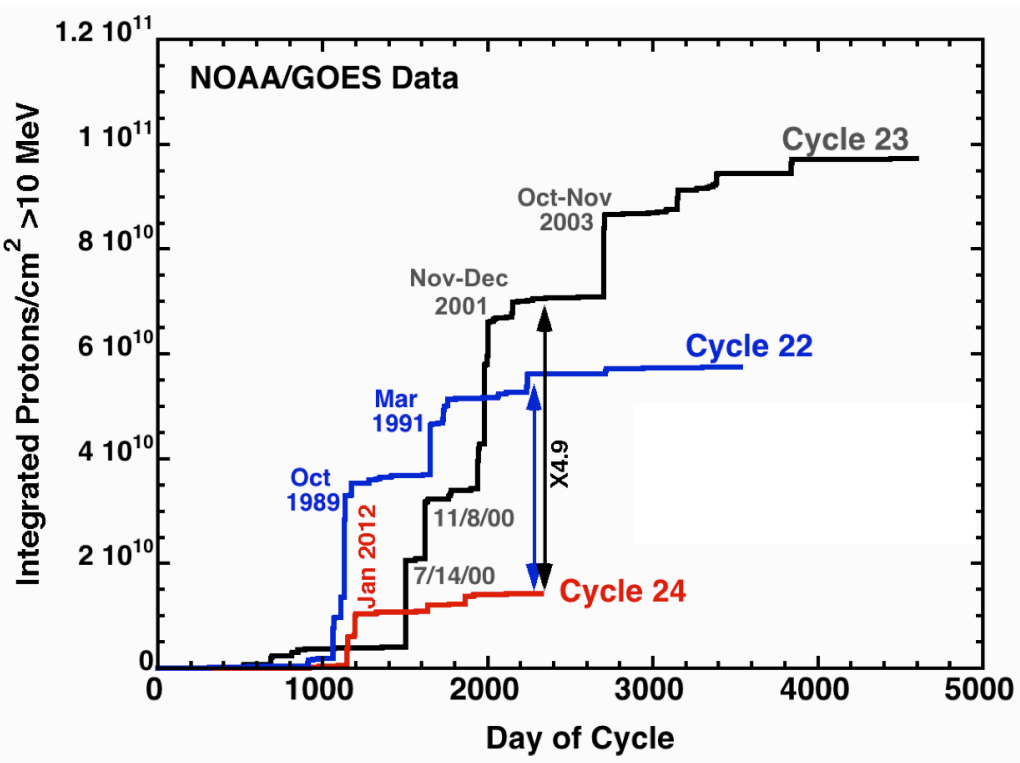

Figure 13: Integral number of energetic protons vs the day of the cycle for cycles 22, 23, and 24 (significant SEP event dates are labeled for each cycle). As of 16 April 2015, cycle 24 was lower than cycles 23 and 22 (at the same point in the cycle) by factors of 4.9 and 4, respectively. From [24].

Given that all of the Severe SEP events occurred in solar cycles 22 and 23, it is surprising that NOAA has recorded no Severe events and only four Strong ones for this cycle (number 24). Although Richardson et al. [21] identified $\sim 185$ SEP events with measureable $>25 \mathrm{MeV}$ protons observed by 1, 2, or 3 spacecraft between 2011 and the end of 2013, it is clear that cycle 24 is lagging behind in SEP production as compared to cycles 22 and 23. Figure 13 compares the integral GOES $>10 \mathrm{MeV}$ proton flux for cycles 22-24 as a function of day of the cycle. As of

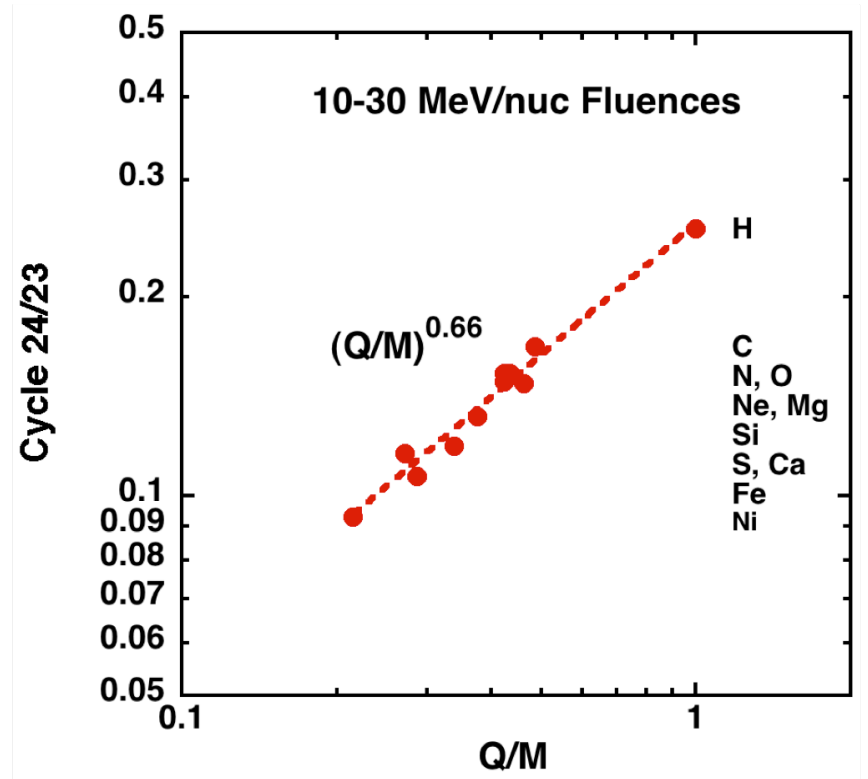

Figure 14: Cycle 24 ion fluences at 10-30 MeV/nuc normalized by those measured in cycle 23 as a function of the element's charge-to-mass ratio $(\mathrm{Q} / \mathrm{M})$ indicating a systematic depletion of the heavy ions in cycle 24. From [24]. 

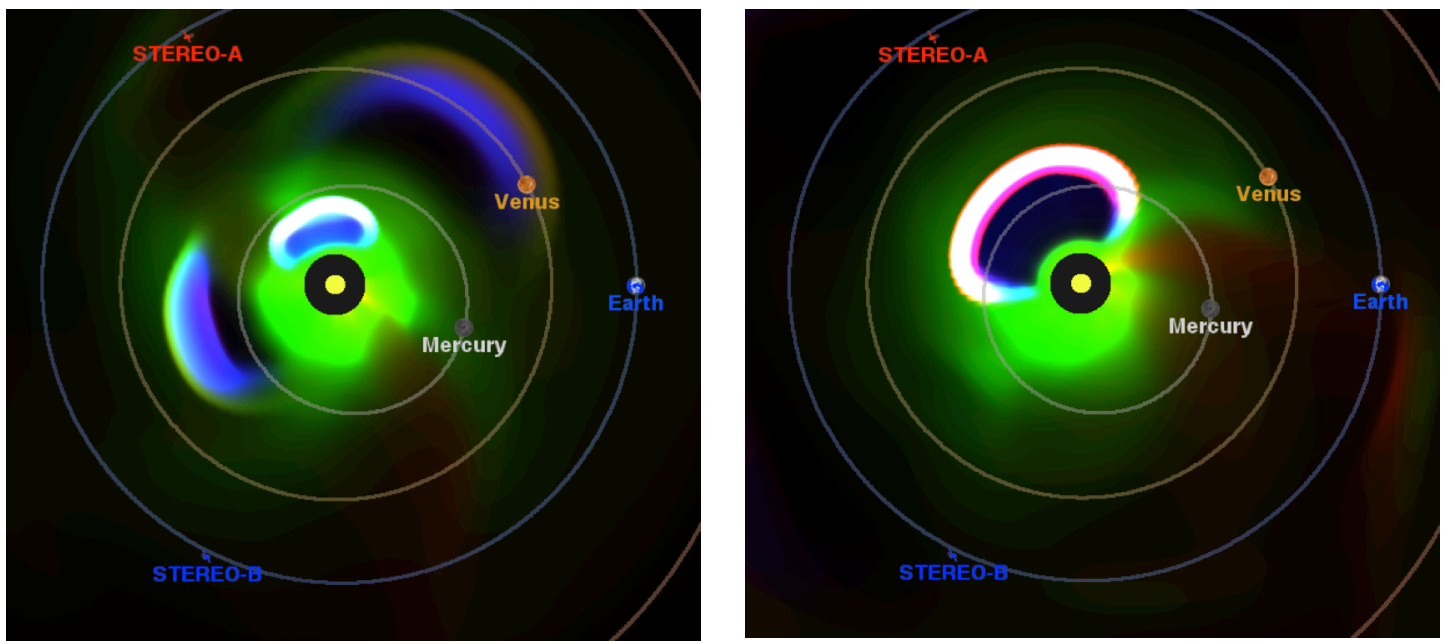

Figure 15: ENLIL simulations of the 23 July 2012 event. (left) Three CMEs occurring in the previous 4 days sweep out a wide range of longitudes, centered on the position of STEREO-AHEAD. (right) The 23 July 2012 CME follows into a rarified interplanetary medium, resulting in little deceleration.

mid-April 2015, the deficit of energetic protons in cycle 24 compared to previous cycles was a factor of 4-5 [24]. The reduction is even stronger when the comparison is made for $>100 \mathrm{MeV}$ protons. Heavy ions are also affected, apparently in a manner governed by the element's ionic charge-to-mass ratio $(\mathrm{Q} / \mathrm{M})$. This can be seen in Figure 14 in which the fluences for $\mathrm{C}-\mathrm{Ni}$ obtained at 10-30 MeV/nuc in cycle 24 are compared to those measured in cycle 23 . The depletions are well organized by $\mathrm{Q} / \mathrm{M}$ with $\mathrm{Ni}$ in cycle 24 being lower by over an order of magnitude. The specific cause of cycle 24's lack of particle production has not yet been determined, but likely candidates are the weaker magnetic field strength and the lower densities of suprathermal particles that are the seed population for SEPs (see [24] for more details).

Despite this weaker cycle, there have been a few significant SEP events. Probably the most notable was the 23 July 2012 event. The source of this event was west of the Sun-Earth line by $140^{\circ}$, located near disk center as viewed by STEREO-A. The emerging CME was so fast that it arrived at STEREO-A in less than 24 hours, with in-situ measured solar wind speeds $>2000 \mathrm{~km} / \mathrm{s}$ and magnetic field strengths of $>100 \mathrm{nT}$, some of the largest values ever measured [25]. Estimates of the geomagnetic impact of such a disturbance, if it had been directed towards Earth instead of STEREO-A, range from the largest geomagnetic storm of the last 25 years to the largest ever recorded [26,27]. Careful 3D modeling of the CME observations obtained by the two STEREO spacecraft and SOHO have allowed the speed, acceleration and evolution of the structure to be determined [28]. From this analysis it is clear that the CME exhibited surprisingly little deceleration on its way to $1 \mathrm{AU}$, most likely due to a preconditioning of the interplanetary medium resulting in unusually low solar wind densities. One possible cause of this was the launch of three successive CMEs over the preceding 4 days which combined to sweep out a large longitudinal section surrounding STEREO-A (Figure 15).

Not surprisingly, such a fast CME drove a shock strong enough to accelerate a large number of SEPs. The time-intensity profile of the SEP event is shown in Figure 16; significant proton intensities at energies up to $100 \mathrm{MeV}$ were observed. When the shock arrived at $1 \mathrm{AU}$, particle intensities at all energies increased simultaneously by factors of 3-20. At Earth, this SEP event was barely seen, with peak $>25 \mathrm{MeV}$ proton intensities more than two orders of 


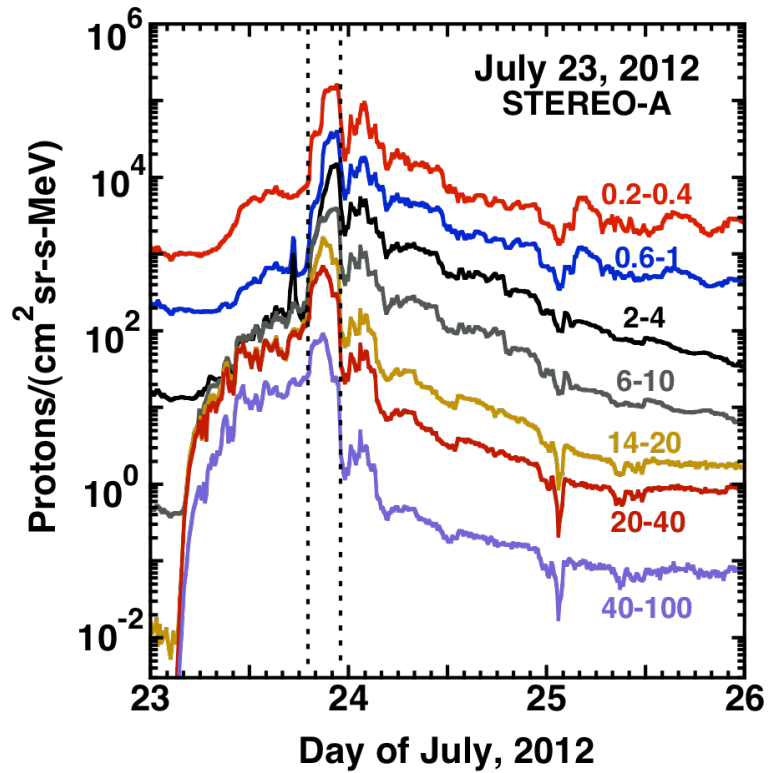

Figure 16: Intensities of protons as several different energy bands vs time as observed by the STEREOA spacecraft for July 232012 SEP event. The dotted lines mark the period in which increases in intensities were observed simultaneously at all energies, due to the arrival of the CME-driven shock at 1 AU. From [30].

magnitude below those observed by STEREO-A [21]. As such, the 23 July 2012 event does not appear on the NOAA list. However, had it been directed at Earth, rather than at STEREO-A, the SEP event would have been classified as Severe and ranked as the third largest recorded, behind only the events of March 1991 and October 1989 [29].

At least one other Severe SEP event was observed in cycle 24, although again only by STEREO-A and missing Earth (and therefore not on NOAA's list). Since August 2014, the small angle between the Sun and the Earth as seen by STEREO-A resulted in unexpected, substantial heating of the high-gain antenna. To alleviate this, the spacecraft pointing has been altered such that communication is limited to using the side lobes of the antenna resulting in significantly reduced telemetry rates. During this time limited data is transmitted for a few hours each day. The uninterrupted data stream for the energetic particle detectors is currently being stored onboard and expectations are that the data will be downloaded once the spacecraft moves significantly around the Sun such that the main lobe of the antenna can be used once more. Unfortunately, the SEP event of 13 December 2014 occurred during this time of limited data transmission. Although the data gaps make it impossible to determine the true peak intensity of the event, it is clear that it was similar in magnitude to that of the 23 July 2012 event (Figure 17). Determination of the actual peak intensity will have to wait until the full dataset is downloaded.

Without the observations being made by the STEREO spacecraft, it would have been erroneously believed that no Severe SEP events occurred (so far) in cycle 24. The Earth was lucky that the 23 July 2012 event did not occur a week earlier when the relevant active region was near $0^{\circ}$ solar longitude. From the STEREO-A observations it is clear that even a cycle considered extremely weak in nearly every aspect (see, e.g., [31,32]) can produce SEP events of substantial space weather concern. 


\section{Lessons Learned and New Puzzles}

With the current fleet of heliospheric missions, the ability to monitor the inner solar system has never been greater. In particular, the STEREO spacecraft allow the Sun and interplanetary space out to $1 \mathrm{AU}$ to be monitored from multiple positions simultaneously, including regions not visible from a near-Earth vantage point. These multi-spacecraft observations have resulted in significant advancements in the studies of solar energetic particle events, particularly their acceleration and transport. Yet, as is often the case when a new capability is obtained or a new regime is explored, more questions than answers have emerged. Not all of them have been detailed here, but a few key ones have.

The ability to monitor the entire Sun, not just the hemisphere visible from Earth, allows the source regions of SEP events to be definitively identified while sophisticated modeling of the multi-spacecraft observations of the associated CMEs provides detailed information regarding the speed, extent, and evolution of these structures. The fraction of beyond-the-limb source regions for SEPs has been found to be $\sim 30 \%$ for events producing $>25 \mathrm{MeV}$ protons. Source regions beyond view from Earth are a clear concern for space weather prediction and the assumption that far removed sources cannot produce a fast onset of SEPs at Earth has been shown to be incorrect. Additional observations of energetic electron and ${ }^{3} \mathrm{He}$-rich events are among the many diverse examples efficient transport over wide longitudes (at times encompassing fully $360^{\circ}$ ) and it remains unclear as to what the responsible mechanism(s) is and/or what interplanetary conditions are required/conducive.

Solar cycle 24 has been shown to be quite different, in many aspects, from previous cycles, including the characteristics of SEP events. The production of $>10 \mathrm{MeV}$ protons in cycle 24 is reduced by factors of 4-5 and is even greater for $>100 \mathrm{MeV}$ protons (factors of 6-7).

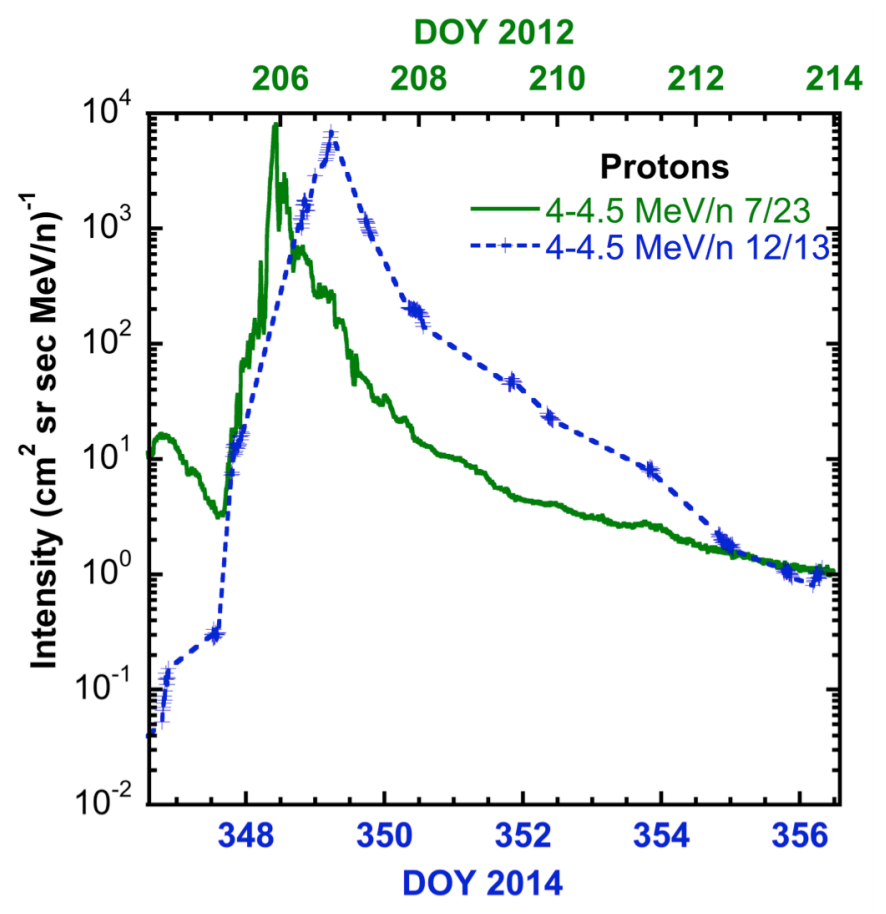

Figure 17: Proton intensities at $\sim 4 \mathrm{MeV}$ vs time for 23 July 2012 (solid green line) and 13 December 2014 (blue plus symbols) SEP events observed by STEREO-A. The December data have significant gaps (dashed blue lines) due to the limited data operations of the spacecraft at this time. 
Additionally there appears to be a further, Q/M-related reduction of heavy ions in SEP events this cycle compared to cycle 23 . It is likely that the reduced magnetic field and lower suprathermal particle densities are contributing factors, but a full explanation remains to be developed. Whether this has significant impacts on expectations of SEP production for upcoming cycles also remains to be seen.

The temptation to be complacent regarding the threat of severe space weather during such a weak cycle would appear to be supported by the near-Earth observations. However, with the STEREO spacecraft it has been made quite clear that mostly Earth has been lucky so far this cycle. At least two severe SEP events have been detected by STEREO-A, one accompanied by a very fast CME which, had it been directed towards Earth, might have created a geomagnetic storm of a severity not experienced for several decades.

The natural next regime of exploration for SEP events and their associated phenomena is the region within $\sim 30 \mathrm{R}_{\mathrm{S}}$ of the Sun. Currently the lack of in-situ measurements well inside 1 AU significantly hampers the understanding of particle acceleration and transport through this region. Fortunately, both the Solar Probe Plus and Solar Orbiter missions plan on exploring this area in the near future. The instrumentation on both spacecraft will produce measurements of solar wind, magnetic fields, and suprathermal and energetic particles which will hopefully lead to answering some of the existing questions regarding SEP acceleration and transport. Undoubtedly they will also reveal additional puzzles that will challenge many aspects of the current understanding; an exciting proposition.

\section{Acknowledgements}

Thank you to NASA's Scientific Visualization Studio, the Space Weather Research Center (SWRC), the Community-Coordinated Modeling Center (CCMC), ENLIL and Dusan Odstrcil (GMU), Leila Mays (CUA) and Janet Luhmann (UCB) and NASA's Scientific Visualization Studio for the images of the ENLIL modeling of the 23 July 2012 event (https://svs.gsfc.nasa.gov/cgi-bin/details.cgi?aid=4167). Images of the 3 November $2011 \mathrm{CME}$ were obtained from Helioviewer.org.

\section{References}

[1] H.V. Cane, R.A. Mewaldt, C.M.S. Cohen, T.T. von Rosenvinge, Role of flares and shocks in determining solar energetic particle abundances, Journal of Geophysical Research 111 (2006) A06S90, doi:10.1029/2005JA011071.

[2] D.V. Reames, Particle acceleration at the Sun and in the heliosphere, Space Science Reviews 90 (1999) 413, doi:10.1023/A:1005105831781.

[3] H.V. Cane, T.T. von Rosenvinge, C.M.S. Cohen, R.A. Mewaldt, Two components in major solar particle events, Geophysical Research Letters 30 (2003) 8017, doi:10.1029/2002GL016580.

[4] A.J. Tylka, C.M.S. Cohen, W.F. Dietrich, M.A. Lee, C.G. Maclennan, R.A. Mewaldt, et al., Shock Geometry, Seed Populations, and the Origin of Variable Elemental Composition at High Energies in Large Gradual Solar Particle Events, The Astrophysical Journal 625 (2005) 474, doi:10.1086/429384. 
[5] A. Driesman, S. Hynes, G. Cancro, The STEREO Observatory, Space Science Reviews 136 (2008) 17, doi:10.1007/s11214-007-9286-z.

[6] M.L. Kaiser, T.A. Kucera, J.M. Davila, O.C. St Cyr, M. Guhathakurta, E. Christian, The STEREO Mission: An Introduction, Space Science Reviews 136 (2008) 5, doi:10.1007/s11214007-9277-0.

[7] J.G. Luhmann, D.W. Curtis, P. Schroeder, J. McCauley, R.P. Lin, D.E. Larson, et al., STEREO IMPACT Investigation Goals, Measurements, and Data Products Overview, Space Science Reviews 136 (2008) 117, doi:10.1007/s11214-007-9170-x.

[8] E.C. Stone, A.M. Frandsen, R.A. Mewaldt, E.R. Christian, D. Margolies, J.F. Ormes, et al., The Advanced Composition Explorer, Space Science Reviews 86 (1998) 1, doi:10.1023/A:1005082526237.

[9] A. Isavnin, A. Vourlidas, E.K.J. Kilpua, Three-Dimensional Evolution of Erupted Flux Ropes from the Sun $\left(2-20 R_{S}\right)$ to 1 AU, Solar Physics 284 (2013) 203, doi:10.1007/s11207012-0214-3.

[10] M.E. Wiedenbeck, G.M. Mason, C.M.S. Cohen, N.V. Nitta, R. Gómez-Herrero, D.K. Haggerty, Observations of Solar Energetic Particles from ${ }^{3}$ He-rich Events over a Wide Range of Heliographic Longitude, The Astrophysical Journal 762 (2013) 54, doi:10.1088/0004$637 \mathrm{X} / 762 / 1 / 54$.

[11] C.M.S. Cohen, M.E. Wiedenbeck, G.M. Mason, R. Gómez-Herrero, D.K. Haggerty, N.V. Nitta, Furthering Our Understanding of Wide Longitude ${ }^{3}$ He-rich SEP Events, in: Proceedings of the 33rd International Cosmic Ray Conference, 2013, paper 0802.

[12] M. Wiedenbeck, G.M. Mason, C.M.S. Cohen, cohen, N.V. Nitta, R. Gómez-Herrero, et al., Observations of Broad Longitudinal Extents of ${ }^{3} \mathrm{He}$-rich SEP Events, in: Proceedings of the 32nd International Cosmic Ray Conference, 2011, 208, doi:10.7529/ICRC2011/V10/1162.

[13] V.S. Titov, Z. Mikić, J.A. Linker, R. Lionello, S.K. Antiochos, Magnetic Topology of Coronal Hole Linkages, The Astrophysical Journal 731 (2011) 111, doi:10.1088/0004$637 \mathrm{X} / 731 / 2 / 111$.

[14] S.K. Antiochos, J.A. Linker, R. Lionello, Z. Mikić, V. Titov, T.H. Zurbuchen, The Structure and Dynamics of the Corona-Heliosphere Connection, Space Science Reviews 172 (2012) 169, doi:10.1007/s11214-011-9795-7.

[15] D.I. Pontin, P.F. Wyper, The Effect of Reconnection on the Structure of the Sun's OpenClosed Flux Boundary, The Astrophysical Journal 805 (2015) 39, doi:10.1088/0004$637 \mathrm{X} / 805 / 1 / 39$.

[16] J. Giacalone, J.R. Jokipii, The Longitudinal Transport of Energetic Ions from Impulsive Solar Flares in Interplanetary Space, The Astrophysical Journal Letters 751 (2012) L33, doi:10.1088/2041-8205/751/2/L33.

[17] C.M.S. Cohen, J. Giacalone, G.M. Mason, M.E. Wiedenbeck, Examining Particle Transport in Multi-Spacecraft ${ }^{3} \mathrm{He}$-rich SEP Events, The Astrophysical Journal, in preparation (2016).

[18] N. Dresing, R. Gómez-Herrero, B. Heber, A. Klassen, O. Malandraki, W. Droge, et al., Statistical survey of widely spread out solar electron events observed with STEREO and ACE with special attention to anisotropies, Astronomy and Astrophysics 567 (2014) A27, doi:10.1051/0004-6361/201423789. 
[19] M.A. Lee, Coupled Hydromagnetic Wave Excitation and Ion Acceleration at an Evolving Coronal/Interplanetary Shock, The Astrophysical Journal Supplement Series 158 (2005) 38, doi:10.1086/428753.

[20] M.A. Lee, Coupled hydromagnetic wave excitation and ion acceleration at interplanetary traveling shocks, Journal of Geophysical Research 88 (1983) 6109, doi:10.1029/JA088iA08p06109.

[21] I.G. Richardson, T.T. von Rosenvinge, H.V. Cane, E.R. Christian, C.M.S. Cohen, A.W. Labrador, et al., > $25 \mathrm{MeV}$ Proton Events Observed by the High Energy Telescopes on the STEREO A and B Spacecraft and/or at Earth During the First $\sim$ Seven Years of the STEREO Mission, Solar Physics 289 (2014) 3059, doi:10.1007/s11207-014-0524-8.

[22] R.A. Mewaldt, C.M.S. Cohen, G.M. Mason, T.T. von Rosenvinge, R.A. Leske, J.G. Luhmann, et al., Solar energetic particles and their variability from the sun and beyond, in: Solar Wind 13: Proceedings of the Thirteenth Solar Wind Conference, AIP, 2013: pp. 116121. doi:10.1063/1.4811002.

[23] H.V. Cane, D.V. Reames, T.T. von Rosenvinge, The role of interplanetary shocks in the longitude distribution of solar energetic particles, Journal of Geophysical Research 93 (1988) 9555, doi:10.1029/JA093iA09p09555.

[24] R.A. Mewaldt, C.M.S. Cohen, G.M. Mason, T.T. von Rosenvinge, G. Li, C.W. Smith, et al., Investigating the Causes of Solar-Cycle Variations in Solar Energetic Particle Fluences and Composition, in: Proceedings of the 34th International Cosmic Ray Conference, 2015, highlight paper.

[25] C.T. Russell, R.A. Mewaldt, J.G. Luhmann, G.M. Mason, T.T. von Rosenvinge, C.M.S. Cohen, et al., The Very Unusual Interplanetary Coronal Mass Ejection of 2012 July 23: A Blast Wave Mediated by Solar Energetic Particles, The Astrophysical Journal 770 (2013) 38, doi:10.1088/0004-637X/770/1/38.

[26] D.N. Baker, X. Li, A. Pulkkinen, C.M. Ngwira, M.L. Mays, A.B. Galvin, et al., A major solar eruptive event in July 2012: Defining extreme space weather scenarios, Space Weather 11 (2013) 585, doi:10.1002/swe.20097.

[27] C.M. Ngwira, A. Pulkkinen, M. Leila Mays, M.M. Kuznetsova, A.B. Galvin, K. Simunac, et al., Simulation of the 23 July 2012 extreme space weather event: What if this extremely rare CME was Earth directed?, Space Weather 11 (2013) 671, doi:10.1002/2013SW000990.

[28] M. Temmer, N.V. Nitta, Interplanetary Propagation Behavior of the Fast Coronal Mass Ejection on 23 July 2012, Solar Physics 290 (2015) 919, doi:10.1007/s11207-014-0642-3.

[29] R.A. Mewaldt, C.M.S. Cohen, R.A. Leske, G.M. Mason, T.T. von Rosenvinge, $A 360^{\circ}$ Survey of Solar Energetic Particle Events and One Extreme Event, in: Proceedings of the 34th International Cosmic Ray Conference, 2015: paper 139.

[30] R.A. Mewaldt, C.T. Russell, C.M.S. Cohen, A.B. Galvin, R. Gómez-Herrero, A. Klassen, et al., A $360^{\circ}$ View of Solar Energetic Particle Events, Including One Extreme Event, in: Proceedings of the 33rd International Cosmic Ray Conference, 2013: paper 1186.

[31] D.J. McComas, N. Angold, H.A. Elliott, G. Livadiotis, N.A. Schwadron, R.M. Skoug, et al., Weakest Solar Wind of the Space Age and the Current "Mini" Solar Maximum, The Astrophysical Journal 779 (2013) 2, doi:10.1088/0004-637X/779/1/2. 
[32] R.A. Mewaldt, A.J. Davis, K.A. Lave, R.A. Leske, E.C. Stone, M.E. Wiedenbeck, et al., Record-setting Cosmic-ray Intensities in 2009 and 2010, The Astrophysical Journal Letters 723 (2010) L1, doi:10.1088/2041-8205/723/1/L1. 